\title{
Detached Eddy Simulation of Flap Side-Edge Flow
}

\author{
Shankar K. Balakrishnan \\ NASA Advanced Supercomputing Division \\ NASA Ames Research Center \\ Moffett Field, CA 94035 \\ shankar.balakrishnan@nasa.gov \\ Karim R. Shariff \\ NASA Advanced Supercomputing Division \\ NASA Ames Research Center \\ Moffett Field, CA 94035 \\ karim.r.shariff@nasa.gov
}

\begin{abstract}
Detached Eddy Simulation (DES) of flap side-edge flow was performed with a wing and half-span flap configuration used in previous experimental and numerical studies. The focus of the study is the unsteady flow features responsible for the production of far-field noise. The simulation was performed at a Reynolds number (based on the main wing chord) of 3.7 million. Reynolds Averaged Navier-Stokes (RANS) simulations were performed as a precursor to the DES. The results of these precursor simulations match previous experimental and RANS results closely. Although the present DES simulations have not reached statistical stationarity yet, some unsteady features of the developing flap side-edge flowfield are presented. In the final paper it is expected that statistically stationary results will be presented including comparisons of surface pressure spectra with experimental data.
\end{abstract}

\section{Introduction}

Increasingly stringent noise regulations at airports drive research efforts to identify the different sources of aircraft noise and develop appropriate noise 
reduction strategies. The noise associated with the flap side-edge flow has been identified as a significant contributor to the overall aircraft noise during landing. The pressure differential at the flap side-edge gives rise to an unsteady vortical flowfield that acts as a source of noise which is effectively scattered by the flap surface to the far field.

Early predictions of airframe noise were based on empirical models. Subsequently experimental studies (Storms et al., 1995; Radeztsky et al., 1998) and numerical simulations (Khorrami et al., 1998; Street, 1998) were performed using part-span flap configurations which provided an understanding of the key aerodynamic characteristics of the mean flow at the side edge. The boundary layer separation at the pressure side of the flap side-edge (near the leading edge) leads to a shear layer which rolls up to form a primary vortex that reattaches on the flap side. Boundary layer separation at the suction side of the flap side-edge forms a weaker secondary vortex. Downstream of the leading edge, the vortices grow in size and undergo a merger to form a single vortex located above the suction side. Further downstream, this merged vortex undergoes a breakdown under certain conditions. The instabilities of the shear layer and the merged vortex, the vortex merger and vortex breakdown were identified as likely noise sources (Khorrami and Singer, 1999). Simplified models of these sources were developed and semi-empirical noise prediction models were proposed.

The present DES aims to further the understanding of the mechanism of noise production at a flap side-edge by obtaining the complete unsteady flowfield. The knowledge gained from this study would useful to develop better noise prediction methods and guide noise mitigation strategies.

\section{Configuration}

The aerodynamic configuration used in this study is a NACA $63_{2}-215$ Mod B wing with a $30 \%$ chord half-span slotted flap. A trailing edge cove is present on the half span of the wing with the flap. This configuration was used in the experimental study conducted in the $7 \times 10 \mathrm{ft}$. wind tunnel at the NASA Ames Research center (Storms et al., 1995). The experiment was performed at a Reynolds number (based on the main wing chord) of 3.7 million and a freestream Mach number of 0.22 at an angle of attack of $10^{\circ}$ and two different flap deflection angles $-29^{\circ}$ and $39^{\circ}$. The $39^{\circ}$ configuration (Figure 1) was chosen for the present simulations since it is closer to an aircraft approach 


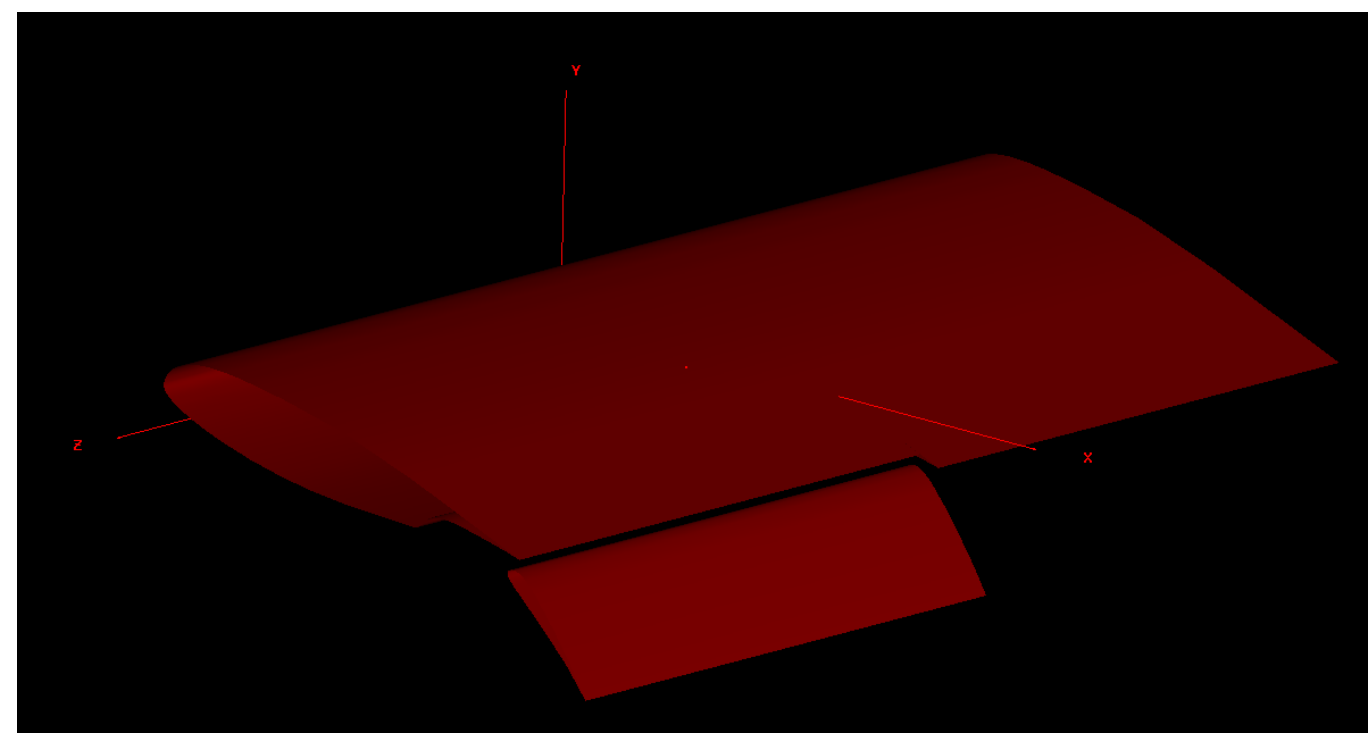

Figure 1: Half span flap configuration used in the present study with a flap deflection of $39^{\circ}$.

configuration at which the flap side-edge noise is most significant.

Acoustic measurements using this configuration were performed at NASA Langley's Quiet Flow Facility (Brooks and Humphreys, 2003) at freestream Mach numbers ranging from 0.07 to 0.17 and Chord based Reynolds numbers 0.7 to $1.7 \times 10^{6}$. Surface pressure measurements at different chordwise locations near the flap side edge were obtained.

\section{Precursor RANS}

As a precursor to the unsteady DES, a RANS simulation was performed using this half-span flap configuration and the setup used in the experiment of Storms et al. (1995). The results of the RANS simulation are compared with the experimental study and a previous thin-layer RANS simulation of the configuration performed by Khorrami et al. (1998) using the SpalartAllmaras model. 


\section{Numerical method}

The Stanford finite-volume based, incompressible Navier-Stokes solver CDP v2.3 (Ham and Iaccarino, 2004; Mahesh et al., 2004) was used for the numerical simulation. The spatial discretization is second-order accurate and the time advancement is performed using a second-order fractional step method. Second order central differencing was used for the viscous terms in the momentum and scalar equations and the first order upwinding scheme was used for the convection terms in both sets of equations. The standard RANS equations were solved and the turbulence model used was the Spalart-Allmaras model.

\section{Setup}

The computational domain and the geometry were chosen to match the experiment (Storms et al., 1995) and the previous RANS (Khorrami et al., 1998). Uniform inflow and extrapolation outflow conditions were applied 15 chord lengths $(c)$ upstream and downstream of the wing leading edge respectively. The wing span and the height of the test-section were 2.25 and 4 chord lengths respectively. The angle of attack was $10^{\circ}$ and the flap deflection was $39^{\circ}$. The Reynolds number based on the wing chord is $3.7 \times 10^{6}$. The wind tunnel walls were treated as free-slip boundaries and the wing and flap surfaces as no-slip boundaries.

A hybrid grid with 37.3 million elements, consisting predominantly of structured cells, was used for the RANS simulation. Figures 2 (a)-(b) contain some images of the grid. The typical wall-normal grid size around the leading edge was $5 \times 10^{-6} c$ equivalent to a $y^{+}$value of approximately 1 .

\section{Comparison with experiment and previous RANS}

Figures $3-4$ present the surface pressure coefficient distributions at two different spanwise locations ( $z=0$ is the midspan location). The PrandtlGlauert corrected results match the results from the experiment and the previous RANS closely. The peak value of $-C_{p}$ on the main wing is nearly the same in all three studies. On the flap the peak $-C_{p}$ is overpredicted by the same amount in both the RANS results compared to the experiment. 


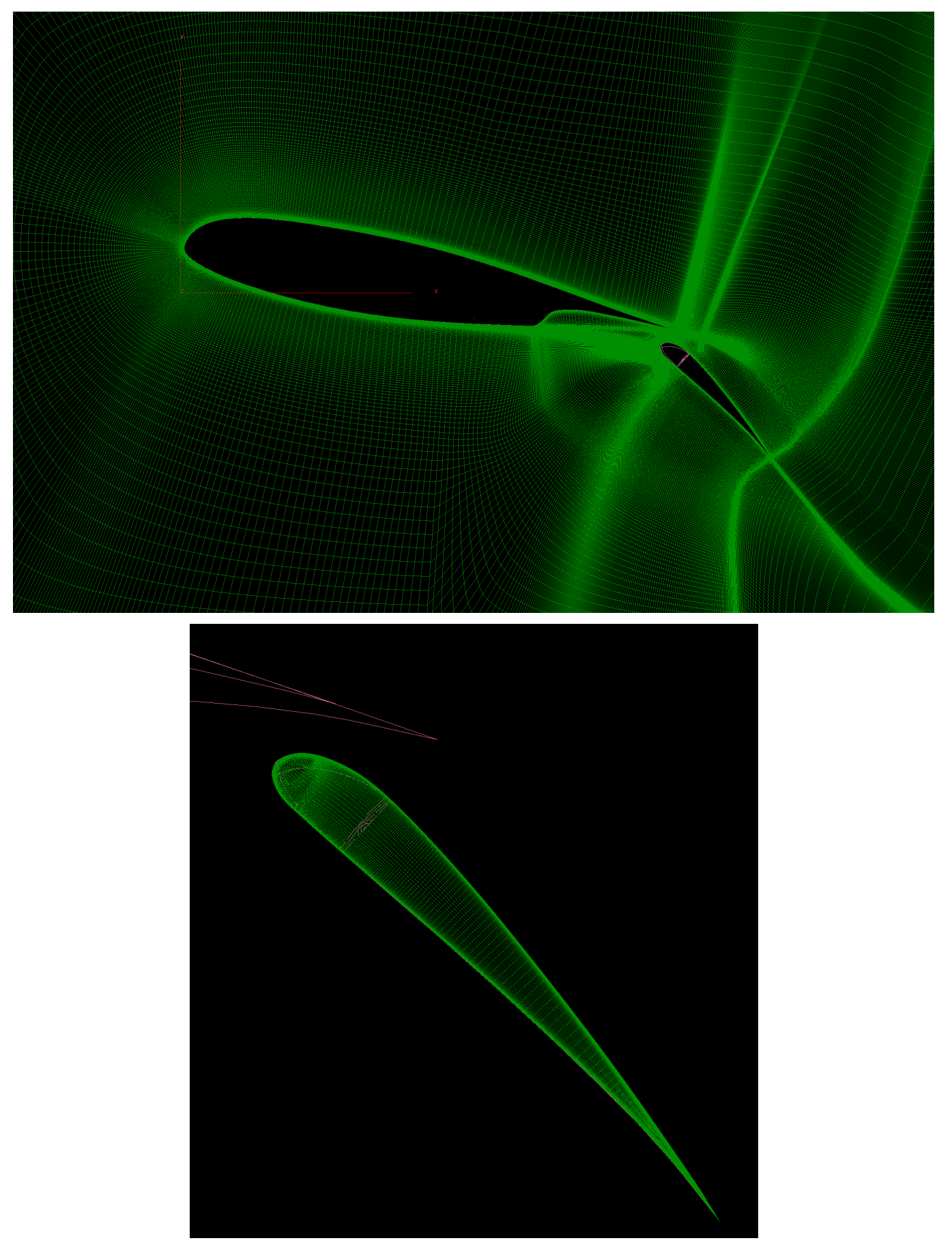

Figure 2: Images of grid used in present RANS. 


\section{Direct Eddy simulation}

The Spalart-Allmaras based delayed-DES (DDES) model (Spalart et al., 2006) is used for the unsteady simulation. The present simulation uses the same numerical schemes for the different terms in the scalar and momentum equations as in the RANS. But future simulations would consider using the central difference scheme for the convection terms of the momentum equation. The converged steady RANS solution was used as the initial condition for the DES.

Figure 5 shows the contours of span-wise vorticity on the mid-span plane (containing the flap side) at the end of two chord flow-through times. An unsteady vortex breakdown is seen to be developing above the flap trailingedge. The final paper will present the various unsteady flow features of the

statistically stationary solution. The unsteady surface pressure spectra would also be compared with the experimental results.

\section{Acknowledgements}

This research is supported by an appointment to the NASA Postdoctoral Program at Ames Research Center, administered by Oak Ridge Associated Universities through a contract with NASA.

\section{References}

Brooks, T. F. and Humphreys, W. M. (2003). Flap-edge aeroacoustic measurements and predictions. Journal of Sound and Vibration, 261(1).

Ham, F. and Iaccarino, G. (2004). Energy conservation in collocated discretization schemes on unstructured meshes. Center of Turbulence Research Annual Research Briefs.

Khorrami, M. R. and Singer, B. A. (1999). Stability analysis for noise-source modeling of a part-span flap. AIAA Journal, 37(10).

Khorrami, M. R., Singer, B. A., and Radeztsky, R. H. (1998). Reynolds averaged navier-stokes computations of a flap side-edge flow field. AIAA 98-0768. 
Mahesh, K., Constantinescu, G., and Moin, P. (2004). A numerical method for large-eddy simulation in complex geometries. J. Comput. Phys., 197.

Radeztsky, R. H., Singer, B. A., and Khorrami, M. R. (1998). Detailed measurements of a flap side-edge flow field. AIAA 98-0700.

Spalart, P. R., Deck, S., Shur, M. L., Shires, K. D., Strelets, M., and Travin, A. (2006). A new version of detached-eddy simulation, resistant to ambiguous grid densities. Theor. Comput. Fluid. Dyn., 20.

Storms, B. L., Takahashi, T. T., and Ross, J. C. (1995). Aerodynamic influence of a finite-span flap on a simple wing. SAE Technical Paper 951977.

Street, C. L. (1998). Numerical simulation of a flap-edge flowfield. AIAA 98-2226. 


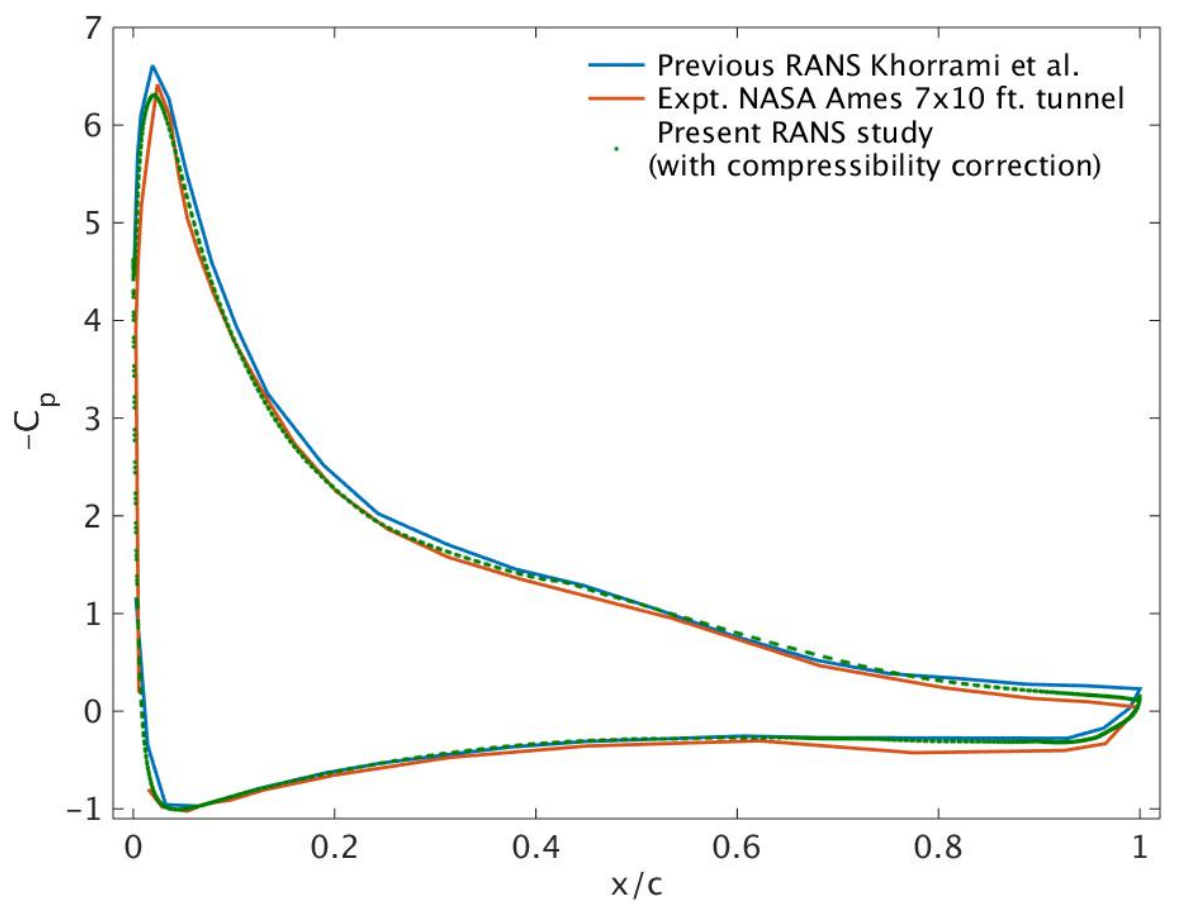

Figure 3: Surface pressure coefficient comparison at spanwise location $z / c=$ -0.660 . 


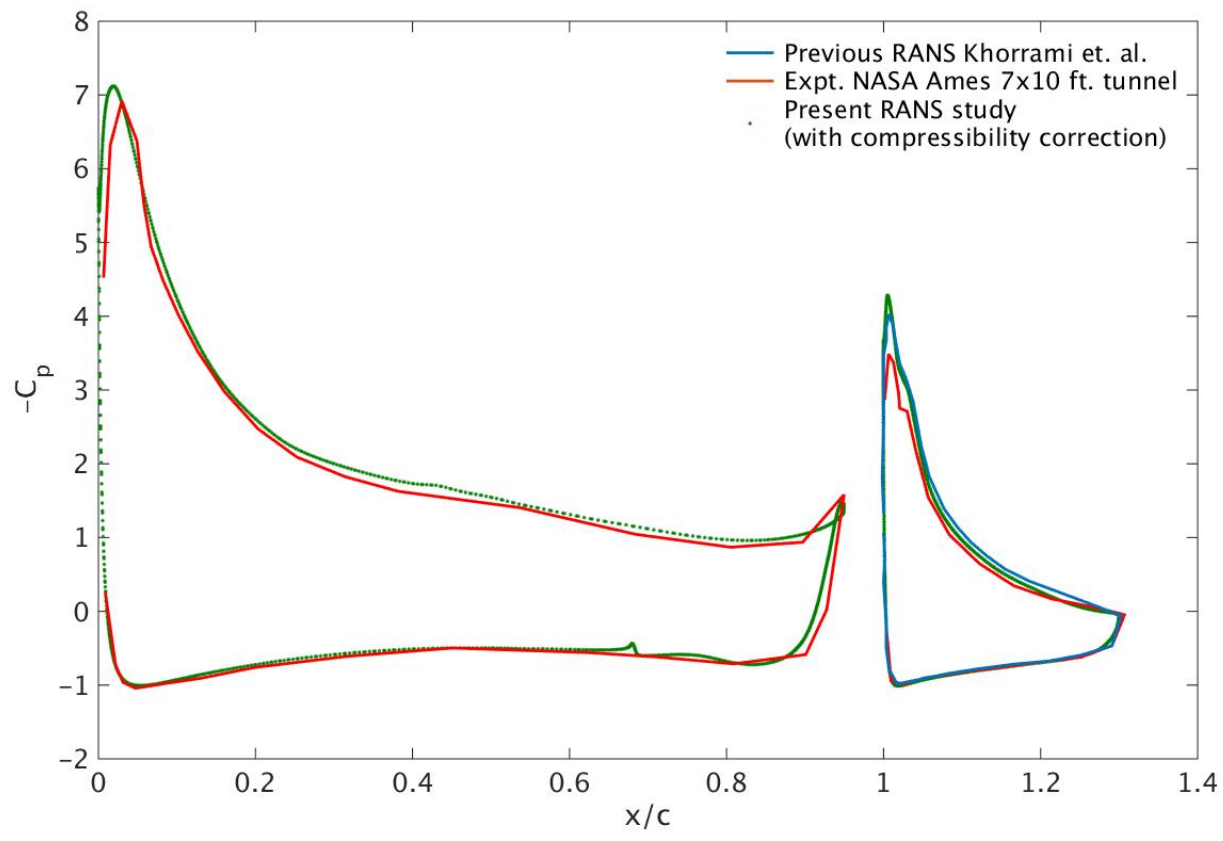

Figure 4: Surface pressure coefficient comparison at spanwise location $z / c=$ 0.440 . 


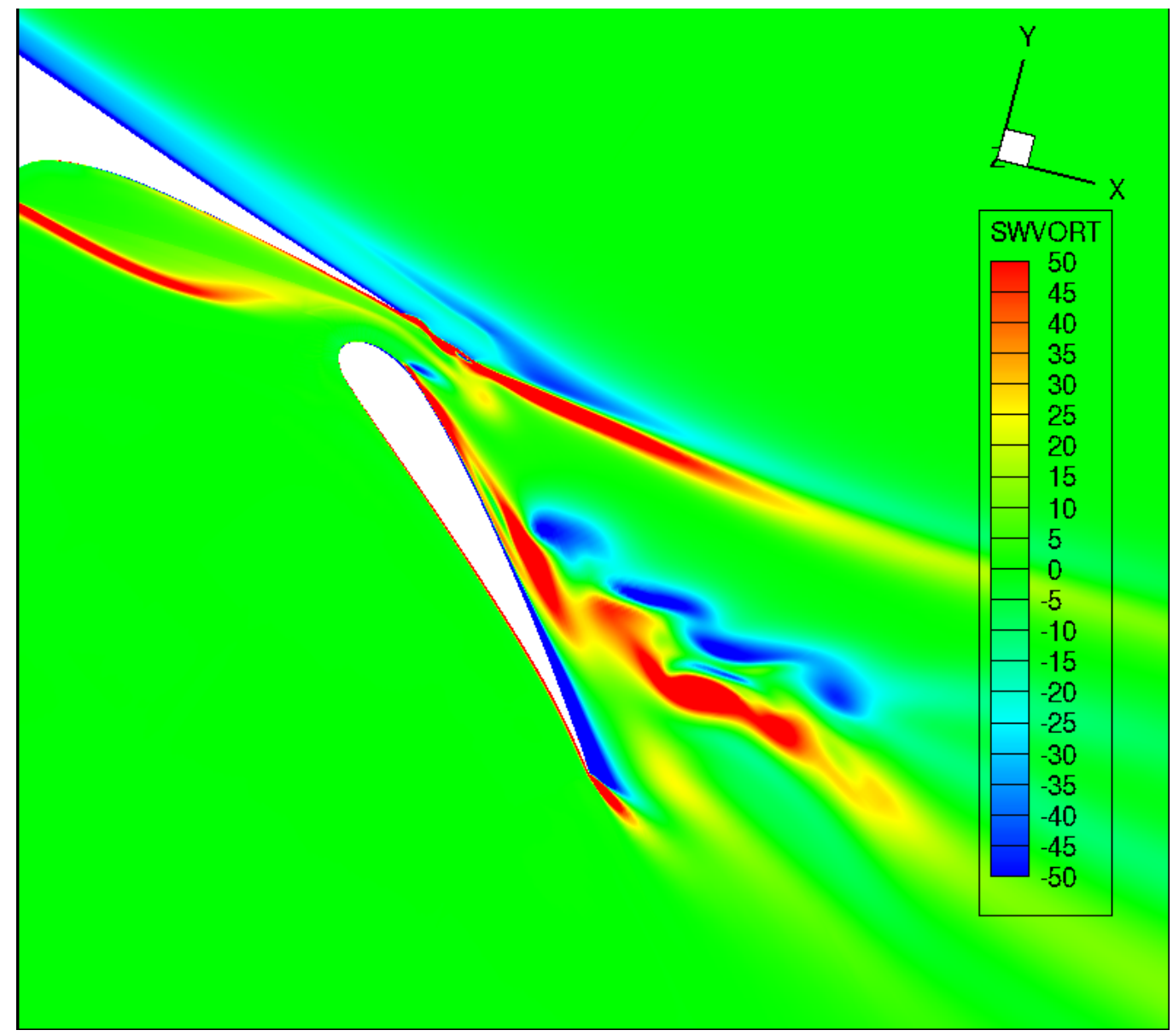

Figure 5: Spanwise Vorticity contours on mid-span plane from developing DES solution. 